\title{
Correlation of maturity groups with seed composition in soybeans, as influenced by genotypic variation
}

\author{
By Damián M. Maestri*, José M. Meriles and Carlos A. Guzmán \\ Cátedra de Química Orgánica, Facultad de Ciencias Exactas, Físicas y Naturales, \\ Universidad Nacional de Córdoba. Av. Vélez Sarsfield 299, Córdoba 5000, Argentina.
}

\section{RESUMEN}

Correlación entre los distintos estados de maduración con la composición de la semilla en soja, influenciada por su variación genotípica.

Se analizaron la humedad, contenido en proteínas, carbohidratos, grasas y cenizas, y las composiciones en ácidos grasos y esteroles de las semillas de 19 cultivares de soja (Glycine max (L.) Merrill) con grupos de madurez V, VI o VII. Los contenidos de proteinas, aceites, carbohidratos y cenizas variaron entre $344-463 \mathrm{~g} \mathrm{~kg}^{-1}, 178-233 \mathrm{~g} \mathrm{~kg}^{-1}$ $234-338 \mathrm{~g} \mathrm{~kg}^{-1}$ y $40.0-49.3 \mathrm{~g} \mathrm{~kg}^{-1}$ de materia seca, respectivamente. Los ácidos grasos mayoritarios fueron palmítico (9.2-12.5\%) oleico (17.7-22.1\%) y linoleico (53.6-56.9\%). El porcentaje de ácido linolénico varió desde 8.6 hasta $10.4 \%$. El principal componente de la fracción de esteroles del aceite fue el sitostero (48.1-56.8\%), seguido por el campesterol (18.4-21.7\%) y el estigmasterol (13.4-18.0\%). Se encontraron diferencias estadísticamente significativas entre los genotipos para la mayoría de los parámetros evaluados, pero no hubo variaciones significativas entre grupos de madurez.

PALABRAS-CLAVE: Ácido graso - Esterol - Genotipo Maduración - Soja (semilla de).

\section{SUMMARY}

Correlation of maturity groups with seed composition in soybeans, as influenced by genotypic variation.

Seeds of 19 soybean cultivars (Glycine max (L.) Merrill) with maturity groups V, VI or VII were analyzed for proximate composition, fatty acids and sterols. Protein, oil, carbohydrate and ash contents varied between $344-463 \mathrm{~g} \mathrm{~kg}^{-1}, 178-233 \mathrm{~g} \mathrm{~kg}^{-1}, 234-338 \mathrm{~g} \mathrm{~kg}^{-1}$, and $40.0-49.3 \mathrm{~g} \mathrm{~kg}^{-1}$ of dry matter, respectively. Fatty acid profiles revealed that the major acids were palmitic $(9.2-12.5 \%)$, oleic $(17.7-22.1 \%)$ and linoleic $(53.6-56.9 \%)$. Linolenic acid ranged from 8.6 to $10.4 \%$. Sitosterol (48.1-56.8\%) was the main component of the sterol fraction, followed by campesterol $(18.4-21.7 \%)$ and stigmasterol (13.4-18.0\%). Statistically significant differences between genotypes were found for the majority of parameters evaluated, but there are not significant variations among maturity groups.

KEY-WORDS: Fatty acid - Genotype - Ripening - Soybean (seed of) - Sterol.

\section{INTRODUCTION}

Expansion of industrial uses of soybeans has stimulated research on composition of the seed, particularly with respect to oil and protein contents and iodine number of oil. As a consequence, the variability of composition has assumed importance, particularly as it is affected by genetic variation, environmental conditions and cultural practices such as date of planting (Carver et al., 1986; Breene et al., 1988; Schnebly and Fehr, 1993). Developing soybean varieties with superior chemical composition to meet special food applications has become a high research priority. During selection of soybeans for a particular food application or a particular seed breeding program, it is important to know the major factors affecting soybean quality such as protein and oil contents, the chemical components of protein and oil, and seed appearance (Liu et al., 1995). Also, there has been an increasing concern about the study of the sterol fraction from seed oils because a specific sterol found in oats, $\Delta^{5}$ - avenasterol, was effective in retarding soybean oil deterioration (Duve and White, 1991).

The present work was undertaken to determine and compare the proximate composition and seed lipid components (fatty acids and sterols) of the most common soybean cultivars grown in Argentina under the same conditions at the Estación Experimental Agropecuaria (EEA-INTA) Manfredi, Córdoba. The broad objetive of our study is to contribute useful chemical information to the genetic quality of germplasm bank materials.

\section{MATERIALS AND METHODS}

\subsection{Plant material}

Nineteen soybean genotypes (Glycine max (L.) Merrill) with maturity groups V, VI or VII were grown 
in 1994/95 at the Estación Experimental Agropecuaria (EEA - INTA, Manfredi, Córdoba, Argentina). Three lots of healthy mature seeds from each cultivar were selected.

\subsection{Proximate analysis}

Moisture, protein, carbohydrate and ash contents were determined by the methods of the AOAC (1980).

\subsection{Oil extraction}

Seeds were milled and extracted with $n$-hexane in a Soxhlet apparatus for 12 hs. After drying the solution with anhydrous sodium sulphate, solvent was removed by vacuum distillation at $40^{\circ} \mathrm{C}$. Oil percentages were determined by weight difference.

\subsection{Fatty acid analysis}

The crude oils were subjected to alkaline saponification (1 N KOH in methanol). Unsaponifiable matter was extracted with n-hexane. The fatty acid methyl esters (FAME) of total lipids were obtained using $1 \mathrm{~N} \mathrm{H}_{2} \mathrm{SO}_{4}$ in methanol and analyzed by gas chromatography (GC) according to Maestri and Guzmán (1993). The theoretical iodine number was calculated according to Carreras et al. (1989).

\subsection{Sterol analysis}

A chloroform solution of unsaponifiable materials was applied uniformly on $20 \times 20 \mathrm{~cm}$ TLC plates coated with $0.5 \mathrm{~mm}$ layer of silica gel and developed with chloroform/ethyl ether $(90: 10, \mathrm{v} / \mathrm{v})$ as eluent (Gaydou et al., 1983). After developing, the plates were sprayed with a solution of rhodamine in ethanol $\left(0.5 \mathrm{~g} \mathrm{litre}^{-1}\right)$ and observed under ultraviolet light. The sterol zones were carefully scraped from the plates and extracted with chloroform for subsequent GC analysis. ACBP ${ }^{-1}$ capillary column was used. Operating conditions were: column temperature, $200-280^{\circ} \mathrm{C}\left(5^{\circ} \mathrm{C}\right.$ $\left.\mathrm{min}^{-1}\right)$; injector and detector temperatures, $300^{\circ} \mathrm{C}$;

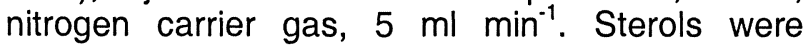
identified by comparing their relative retention times with those of authentic samples and those published by Padley et al. (1986).

\subsection{Statistical analyses}

All chemical determinations were conducted in triplicate. Statistical differences were estimated from one-way ANOVA test at the $5 \%$ level $(P=0.05)$ of significance for all parameters evaluated. Least significant difference (LSD) was performed to establish relationships between each of two variables tested.

\section{RESULTS AND DISCUSSION}

Proximate analyses of the 19 cultivars investigated are shown in Table I. The NK 555, Asgrow 6404 and RA 702 cultivars have high protein content $(444,461$ and $463 \mathrm{~g} \mathrm{~kg}^{-1}$, respectively); while Federada 1, Hood 75, RA 587, Torcaza 63, Copetona 53 and Prata cultivars present an oil content higher than $220 \mathrm{~g}$ $\mathrm{kg}^{-1}$. The carbohydrate contents constitute more than $230 \mathrm{~g} \mathrm{~kg}^{-1}$ in all samples, reaching values of $338 \mathrm{~g}$ $\mathrm{kg}^{-1}$ in Torcacita 58 cultivar. The ash contents range from 40.0 to $49.3 \mathrm{~g} \mathrm{~kg}^{-1}$. Comparison of the average values of oil content in each maturity group shows the highest values for group VI. Mean values for protein, carbohydrate and ash contents are nearly the same. Although more data are needed to demonstrate whether there is an effect of maturity group on both protein and oil contents, there is no indication of an inverse relationship between them as suggested by Liu et al. (1995).

The fatty acid compositions (Table II) are characterized by a high proportion of unsaturated fatty acids which vary between $73.1-87.2 \%$. Palmitic acid is the main saturated acid in all cultivars ranging from 9.2 to $12.5 \%$. Among the unsaturated fatty acids, linoleic is the principal constituent (53.6-56.9\%), followed by oleic (17.7-22.1\%) and linolenic (8.6-10.4\%) acids. The RA 587, Federada 1, Tacuarí and Hood 75 cultivars show the lowest linolenic acid contents. The average value of oleic acid content in maturity group $\mathrm{VI}$ is the highest. There are not significant variations among maturity groups for palmitic, stearic, linoleic and linolenic acids. Cultivars vary considerably in IVs (139.5-147.0); RA 587 has the smallest (Table I). When mean values in each maturity group are considered, no significant differences are found between them for both parameters mentioned.

The following 4-desmethylsterols were detected: cholesterol, campesterol, stigmasterol, $\beta$-sitosterol, $\Delta^{5}$-avenasterol, $\Delta^{7}$-stigmastenol and $\Delta^{7}$-avenasterol (Table III). These results are in general agreement with those previously published by Padley et al. (1986). The $\beta$-sitosterol, campesterol and stigmasterol are the principal constituents, with $\beta$-sitosterol dominant in all samples (48.1-56.8\%). An interesting feature is the presence of larger amounts of $\Delta^{5}$-avenasterol in Montera 74 and Charata 76 cultivars. This sterol was found to be an effective agent at reducing changes in the soybean oil during heating (Duve and White, 1991). On the other hand, cholesterol is present in small quantities (trace-0.9\%). 
Table 1

Moisture $\left(\mathrm{g} \mathrm{kg}^{-1}\right)$, protein, oil, carbohydrate (Carb) and ash contents ( $\mathrm{g} \mathrm{kg}^{-1}$ of dry matter) of seeds, and refractive indices (RI) and iodine values (IV) of the oils from 19 soybean cultivars. Mean values \pm standard deviations, $n=3$.

\begin{tabular}{|c|c|c|c|c|c|c|c|}
\hline Cultivar & Moisture & Protein & Oil & Carb & RI & IV & Ash \\
\hline \multicolumn{8}{|l|}{ Maturity group V } \\
\hline NK 555 & $45.7 \pm 0.5$ & $444 \pm 0.7$ & $192 \pm 0.6$ & $272 \pm 0.4$ & $1.463 \pm 0.03$ & $143.4 \pm 0.9$ & $45.8 \pm 0.2$ \\
\hline Torcacita 58 & $70.3 \pm 0.4$ & $344 \pm 0.8$ & $200 \pm 0.5$ & $338 \pm 0.4$ & $1.466 \pm 0.04$ & $143.6 \pm 0.8$ & $47.1 \pm 0.3$ \\
\hline Asgrow 5308 & $68.3 \pm 0.5$ & $379 \pm 0.7$ & $219 \pm 0.4$ & $285 \pm 0.3$ & $1.460 \pm 0.05$ & $142.9 \pm 0.7$ & $48.6 \pm 0.4$ \\
\hline Asgrow 5409 & $44.4 \pm 0.4$ & $367 \pm 0.6$ & $218 \pm 0.5$ & $333 \pm 0.5$ & $1.468 \pm 0.05$ & $144.0 \pm 0.8$ & $40.3 \pm 0.3$ \\
\hline Tancacha & $47.6 \pm 0.5$ & $389 \pm 0.7$ & $209 \pm 0.6$ & $308 \pm 0.4$ & $1.464 \pm 0.04$ & $144.2 \pm 0.9$ & $46.3 \pm 0.5$ \\
\hline RA 587 & $72.0 \pm 0.4$ & $416 \pm 0.6$ & $222 \pm 0.5$ & $240 \pm 0.5$ & $1.469 \pm 0.05$ & $139.5 \pm 0.8$ & $49.3 \pm 0.4$ \\
\hline Copetona 53 & $69.8 \pm 0.6$ & $395 \pm 0.8$ & $233 \pm 0.4$ & $262 \pm 0.4$ & $1.466 \pm 0.06$ & $148.8 \pm 0.7$ & $40.0 \pm 0.4$ \\
\hline Federada Casilda & $44.7 \pm 0.5$ & $401 \pm 0.7$ & $194 \pm 0.5$ & $316 \pm 0.3$ & $1.468 \pm 0.05$ & $146.7 \pm 0.8$ & $44.3 \pm 0.4$ \\
\hline Tacuarí & $43.8 \pm 0.5$ & $411 \pm 0.6$ & $208 \pm 0.4$ & $288 \pm 0.3$ & $1.468 \pm 0.05$ & $144.3 \pm 0.8$ & $48.3 \pm 0.3$ \\
\hline \multicolumn{8}{|l|}{ Maturity groups VI } \\
\hline NK 641 & $70.3 \pm 0.5$ & $370 \pm 0.7$ & $206 \pm 0.6$ & $309 \pm 0.5$ & $1.468 \pm 0.04$ & $143.8 \pm 0.7$ & $44.5 \pm 0.3$ \\
\hline Federada 1 & $70.3 \pm 0.6$ & $377 \pm 0.6$ & $221 \pm 0.5$ & $288 \pm 0.3$ & $1.472 \pm 0.03$ & $143.1 \pm 0.6$ & $43.7 \pm 0.2$ \\
\hline Hood 75 & $71.5 \pm 0.4$ & $384 \pm 0.6$ & $223 \pm 0.4$ & $272 \pm 0.4$ & $1.470 \pm 0.02$ & $143.0 \pm 0.7$ & $49.0 \pm 0.3$ \\
\hline Asgrow 6404 & $49.5 \pm 0.5$ & $461 \pm 0.5$ & $207 \pm 0.5$ & $234 \pm 0.4$ & $1.467 \pm 0.04$ & $145.5 \pm 0.8$ & $48.1 \pm 0.4$ \\
\hline Torcaza 63 & $69.2 \pm 0.6$ & $404 \pm 0.6$ & $223 \pm 0.4$ & $260 \pm 0.3$ & $1.469 \pm 0.04$ & $141.3 \pm 0.7$ & $43.7 \pm 0.4$ \\
\hline Prata & $47.1 \pm 0.5$ & $389 \pm 0.7$ & $221 \pm 0.5$ & $300 \pm 0.4$ & $1.465 \pm 0.05$ & $145.8 \pm 0.6$ & $42.9 \pm 0.3$ \\
\hline \multicolumn{8}{|l|}{ Maturity group VII } \\
\hline Montera 74 & $65.2 \pm 0.5$ & $404 \pm 0.7$ & $200 \pm 0.3$ & $286 \pm 0.4$ & $1.464 \pm 0.04$ & $146.0 \pm 0.8$ & $44.2 \pm 0.3$ \\
\hline Granera 73 & $46.4 \pm 0.4$ & $388 \pm 0.8$ & $212 \pm 0.4$ & $309 \pm 0.3$ & $1.469 \pm 0.03$ & $147.0 \pm 0.7$ & $44.2 \pm 0.5$ \\
\hline RA 702 & $46.5 \pm 0.4$ & $463 \pm 0.9$ & $178 \pm 0.2$ & $266 \pm 0.3$ & $1.465 \pm 0.05$ & $145.6 \pm 0.5$ & $45.9 \pm 0.3$ \\
\hline Charata 76 & $43.0 \pm 0.5$ & $384 \pm 0.7$ & $191 \pm 0.4$ & $334 \pm 0.4$ & $1.462 \pm 0.04$ & $145.5 \pm 0.6$ & $47.9 \pm 0.4$ \\
\hline
\end{tabular}

Table II

Fatty acid composition (\% of total fatty acids) and oleic to linolenic $(O / L n)$ ratios of seed oils from 19 soybean cultivars. Mean values \pm standard deviations, $n=3$.

\begin{tabular}{|c|c|c|c|c|c|c|c|c|c|c|c|}
\hline Cultivar & $14: 0$ & $16: 0$ & $16: 1$ & $18: 0$ & $18: 1$ & $18: 2$ & $18: 3$ & $20: 0$ & $20: 1$ & $22: 0$ & $O / L n$ \\
\hline \multicolumn{12}{|l|}{ Maturity group V } \\
\hline NK 555 & $t^{n}$ & $11.5 \pm 0.2$ & $\operatorname{tr}$ & $4.0 \pm 0.1$ & $19.2 \pm 0.3$ & $54.9 \pm 0.4$ & $9.6 \pm 0.1$ & $0.5 \pm 0.1$ & $0.2 \pm 0.1$ & $\operatorname{tr}$ & $1.99 \pm 0.1$ \\
\hline Torcacita 58 & $\operatorname{tr}$ & $11.1 \pm 0.2$ & $\operatorname{tr}$ & $3.9 \pm 0.1$ & $20.1 \pm 0.4$ & $55.8 \pm 0.6$ & $8.9 \pm 0.2$ & $\operatorname{tr}$ & $\operatorname{tr}$ & $\operatorname{tr}$ & $2.25 \pm 0.2$ \\
\hline Asgrow 5308 & $\operatorname{tr}$ & $11.1 \pm 0.1$ & $\operatorname{tr}$ & $3.7 \pm 0.2$ & $21.0 \pm 0.3$ & $54.4 \pm 0.7$ & $9.2 \pm 0.1$ & $0.2 \pm 0.1$ & $0.2 \pm 0.1$ & $\operatorname{tr}$ & $2.27 \pm 0.2$ \\
\hline Asgrow 5409 & $\operatorname{tr}$ & $9.2 \pm 0.1$ & $\operatorname{tr}$ & $4.5 \pm 0.1$ & $20.6 \pm 0.5$ & $54.8 \pm 0.7$ & $9.5 \pm 0.2$ & $0.6 \pm 0.2$ & $0.3 \pm 0.1$ & $0.3 \pm 0.1$ & $2.17 \pm 0.1$ \\
\hline Tancacha & $\operatorname{tr}$ & $10.5 \pm 0.1$ & $\operatorname{tr}$ & $3.4 \pm 0.2$ & $22.1 \pm 0.5$ & $54.7 \pm 0.8$ & $9.2 \pm 0.2$ & $\operatorname{tr}$ & $\operatorname{tr}$ & $\operatorname{tr}$ & $2.40 \pm 0.2$ \\
\hline RA 587 & $\operatorname{tr}$ & $11.7 \pm 0.2$ & $\operatorname{tr}$ & $3.1 \pm 0.2$ & $21.0 \pm 0.4$ & $53.6 \pm 0.7$ & $8.6 \pm 0.3$ & $\operatorname{tr}$ & $\operatorname{tr}$ & $\operatorname{tr}$ & $2.44 \pm 0.3$ \\
\hline Copetona 53 & $\operatorname{tr}$ & $10.3 \pm 0.3$ & $\operatorname{tr}$ & $1.6 \pm 0.1$ & $20.3 \pm 0.6$ & $56.5 \pm 0.9$ & $10.2 \pm 0.2$ & $0.3 \pm 0.1$ & $0.2 \pm 0.2$ & $0.4 \pm 0.2$ & $1.99 \pm 0.2$ \\
\hline Federada Casilda & $\operatorname{tr}$ & $10.6 \pm 0.2$ & $\operatorname{tr}$ & $4.1 \pm 0.3$ & $18.5 \pm 0.7$ & $56.5 \pm 0.8$ & $10.1 \pm 0.3$ & $\operatorname{tr}$ & $\operatorname{tr}$ & tr & $1.83 \pm 0.2$ \\
\hline Tacuari & $0.1 \pm 0.1$ & $10.9 \pm 0.3$ & $0.1 \pm 0.1$ & $3.6 \pm 0.4$ & $19.1 \pm 0.6$ & $56.7 \pm 0.8$ & $8.8 \pm 0.2$ & $0.2 \pm 0.1$ & $0.2 \pm 0.1$ & $\operatorname{tr}$ & $2.17 \pm 0.3$ \\
\hline \multicolumn{12}{|l|}{ Maturity groups VI } \\
\hline NK 641 & $\operatorname{tr}$ & $9.8 \pm 0.2$ & $\operatorname{tr}$ & $4.3 \pm 0.1$ & $20.8 \pm 0.4$ & $55.1 \pm 0.6$ & $9.1 \pm 0.3$ & $0.3 \pm 0.1$ & $0.2 \pm 0.1$ & $0.3 \pm 0.2$ & $2.28 \pm 0.4$ \\
\hline Federada 1 & $\operatorname{tr}$ & $12.5 \pm 0.2$ & $0.6 \pm 0.1$ & $3.8 \pm 0.1$ & $17.7 \pm 0.5$ & $56.6 \pm 0.8$ & $8.8 \pm 0.2$ & $\operatorname{tr}$ & $\operatorname{tr}$ & $\operatorname{tr}$ & $2.01 \pm 0.3$ \\
\hline Hood 75 & $\operatorname{tr}$ & $11.8 \pm 0.3$ & $0.1 \pm 0.1$ & $3.0 \pm 0.2$ & $20.5 \pm 0.4$ & $55.3 \pm 0.7$ & $8.8 \pm 0.2$ & $0.1 \pm 0.1$ & $0.1 \pm 0.1$ & tr & $2.32 \pm 0.2$ \\
\hline Asgrow 6404 & $\operatorname{tr}$ & $10.5 \pm 0.2$ & $\operatorname{tr}$ & $3.6 \pm 0.3$ & $18.0 \pm 0.4$ & $55.9 \pm 0.8$ & $10.2 \pm 0.1$ & $\operatorname{tr}$ & $\operatorname{tr}$ & $\operatorname{tr}$ & $1.76 \pm 0.3$ \\
\hline Torcaza 63 & $\operatorname{tr}$ & $10.6 \pm 0.2$ & $\operatorname{tr}$ & $4.6 \pm 0.1$ & $20.8 \pm 0.4$ & $53.7 \pm 0.9$ & $9.1 \pm 0.3$ & $0.3 \pm 0.1$ & $0.4 \pm 0.1$ & $0.4 \pm 0.1$ & $2.28 \pm 0.4$ \\
\hline Prata & $0.3 \pm 0.1$ & $10.9 \pm 0.4$ & $0.5 \pm 0.1$ & $3.8 \pm 0.1$ & $18.4 \pm 0.3$ & $55.7 \pm 0.8$ & $10.1 \pm 0.2$ & $0.1 \pm 0.1$ & $0.1 \pm 0.1$ & $\operatorname{tr}$ & $1.82 \pm 0.3$ \\
\hline \multicolumn{12}{|l|}{ Maturity group VII } \\
\hline Montera 74 & $0.3 \pm 0.1$ & $9.8 \pm 0.4$ & $0.4 \pm 0.1$ & $4.4 \pm 0.2$ & $19.5 \pm 0.3$ & $55.4 \pm 0.8$ & $10.1 \pm 0.2$ & $\operatorname{tr}$ & $\operatorname{tr}$ & $\operatorname{tr}$ & $1.93 \pm 0.4$ \\
\hline Granera 73 & $0.1 \pm 0.1$ & $9.8 \pm 0.3$ & $0.3 \pm 0.1$ & $3.5 \pm 0.2$ & $20.2 \pm 0.4$ & $56.1 \pm 0.7$ & $9.8 \pm 0.3$ & $\operatorname{tr}$ & $\operatorname{tr}$ & $\operatorname{tr}$ & $2.06 \pm 0.3$ \\
\hline RA 702 & $\operatorname{tr}$ & $11.1 \pm 0.2$ & $0.1 \pm 0.1$ & $3.4 \pm 0.2$ & $18.9 \pm 0.4$ & $56.9 \pm 0.9$ & $9.2 \pm 0.2$ & $0.2 \pm 0.1$ & $\operatorname{tr}$ & $\operatorname{tr}$ & $2.04 \pm 0.4$ \\
\hline Charata 76 & $\operatorname{tr}$ & $10.2 \pm 0.3$ & $\operatorname{tr}$ & $3.3 \pm 0.1$ & $19.3 \pm 0.3$ & $54.7 \pm 0.8$ & $10.4 \pm 0.2$ & $0.1 \pm 0.1$ & $0.6 \pm 0.2$ & $1.0 \pm 0.2$ & $1.85 \pm 0.4$ \\
\hline
\end{tabular}

${ }^{a}$ tr, trace: $<0.1 \%$ 
Table III

Sterol $^{\mathrm{a}}$ composition (\% of total sterols) of seed oils from 19 soybean cultivars. Mean values \pm standard deviations, $n=3$.

\begin{tabular}{|c|c|c|c|c|c|c|c|}
\hline Cultivar & Chol & Camp & Stig & $\beta$-Sit & $\Delta^{5}-A v$ & $\Delta^{7}$-Stig & $\Delta^{7}-A v$ \\
\hline \multicolumn{8}{|l|}{ Maturity group V } \\
\hline NK 555 & $0.6 \pm 0.1$ & $19.8 \pm 0.4$ & $14.8 \pm 0.3$ & $55.9 \pm 0.7$ & $4.0 \pm 0.2$ & $3.4 \pm 0.1$ & $1.3 \pm 0.1$ \\
\hline Torcacita 58 & $0.5 \pm 0.1$ & $18.4 \pm 0.3$ & $15.6 \pm 0.2$ & $55.0 \pm 0.8$ & $5.2 \pm 0.2$ & $3.6 \pm 0.2$ & $1.6 \pm 0.1$ \\
\hline Asgrow 5308 & $\operatorname{tr}^{b}$ & $20.5 \pm 0.3$ & $14.3 \pm 0.2$ & $51.2 \pm 0.9$ & $6.5 \pm 0.1$ & $4.4 \pm 0.2$ & $1.6 \pm 0.1$ \\
\hline Asgrow 5409 & $\operatorname{tr}$ & $21.7 \pm 0.2$ & $18.0 \pm 0.3$ & $48.3 \pm 0.8$ & $4.4 \pm 0.2$ & $3.1 \pm 0.1$ & $1.1 \pm 0.1$ \\
\hline Tancacha & $\operatorname{tr}$ & $18.7 \pm 0.2$ & $15.9 \pm 0.3$ & $55.2 \pm 0.7$ & $5.4 \pm 0.1$ & $2.9 \pm 0.1$ & $1.6 \pm 0.1$ \\
\hline RA 587 & $0.7 \pm 0.1$ & $20.9 \pm 0.4$ & $15.8 \pm 0.3$ & $51.1 \pm 0.7$ & $5.8 \pm 0.2$ & $3.8 \pm 0.1$ & $1.8 \pm 0.2$ \\
\hline Copetona 53 & $0.4 \pm 0.1$ & $21.1 \pm 0.3$ & $15.8 \pm 0.2$ & $51.8 \pm 0.9$ & $5.6 \pm 0.2$ & $4.2 \pm 0.2$ & $1.0 \pm 0.2$ \\
\hline Federada Casilda & $0.9 \pm 0.1$ & $20.5 \pm 0.4$ & $16.2 \pm 0.3$ & $52.1 \pm 0.8$ & $5.8 \pm 0.1$ & $3.6 \pm 0.2$ & $0.8 \pm 0.1$ \\
\hline Tacuarí & $\operatorname{tr}$ & $18.7 \pm 0.3$ & $15.9 \pm 0.2$ & $53.0 \pm 0.7$ & $6.3 \pm 0.2$ & $5.4 \pm 0.1$ & $0.6 \pm 0.2$ \\
\hline \multicolumn{8}{|l|}{ Maturity groups VI } \\
\hline NK 641 & $0.7 \pm 0.1$ & $19.4 \pm 0.4$ & $14.5 \pm 0.5$ & $53.2 \pm 0.9$ & $6.1 \pm 0.1$ & $4.9 \pm 0.2$ & $1.1 \pm 0.1$ \\
\hline Federada 1 & $0.4 \pm 0.1$ & $21.0 \pm 0.3$ & $14.4 \pm 0.4$ & $54.2 \pm 0.7$ & $4.5 \pm 0.2$ & $4.0 \pm 0.1$ & $1.4 \pm 0.1$ \\
\hline Hood 75 & $\operatorname{tr}$ & $19.4 \pm 0.3$ & $15.1 \pm 0.2$ & $54.4 \pm 0.8$ & $5.6 \pm 0.1$ & $3.4 \pm 0.2$ & $2.0 \pm 0.2$ \\
\hline Asgrow 6404 & $0.9 \pm 0.2$ & $18.5 \pm 0.4$ & $13.4 \pm 0.6$ & $56.8 \pm 0.9$ & $6.1 \pm 0.1$ & $3.1 \pm 0.1$ & $1.1 \pm 0.1$ \\
\hline Torcaza 63 & $\operatorname{tr}$ & $20.0 \pm 0.3$ & $17.3 \pm 0.4$ & $48.1 \pm 0.7$ & $5.5 \pm 0.2$ & $3.8 \pm 0.2$ & $1.4 \pm 0.1$ \\
\hline Prata & $0.8 \pm 0.1$ & $19.8 \pm 0.3$ & $15.5 \pm 0.5$ & $52.2 \pm 0.8$ & $6.3 \pm 0.1$ & $4.1 \pm 0.1$ & $1.2 \pm 0.1$ \\
\hline \multicolumn{8}{|l|}{ Maturity group VII } \\
\hline Montera 74 & $0.8 \pm 0.2$ & $18.4 \pm 0.4$ & $14.3 \pm 0.3$ & $55.1 \pm 0.9$ & $6.9 \pm 0.2$ & $3.7 \pm 0.2$ & $0.7 \pm 0.1$ \\
\hline Granera 73 & tr & $19.7 \pm 0.3$ & $17.3 \pm 0.3$ & $50.2 \pm 0.9$ & $6.3 \pm 0.1$ & $4.5 \pm 0.1$ & $1.9 \pm 0.1$ \\
\hline RA 702 & $\operatorname{tr}$ & $18.9 \pm 0.5$ & $17.0 \pm 0.4$ & $51.4 \pm 0.8$ & $5.1 \pm 0.1$ & $4.8 \pm 0.2$ & $2.1 \pm 0.1$ \\
\hline Charata 76 & $0.7 \pm 0.1$ & $20.7 \pm 0.4$ & $16.0 \pm 0.3$ & $50.8 \pm 0.7$ & $6.5 \pm 0.1$ & $4.0 \pm 0.2$ & $1.2 \pm 0.1$ \\
\hline
\end{tabular}

${ }^{a}$ Chol, cholesterol; Camp, campesterol; Stig, stigmasterol; $\beta$-Sit, $\beta$-sitosterol; $\Delta^{5}-A v, \Delta^{5}$-avenasterol; $\Delta^{7}$-Sitg, $\Delta^{7}$-Stigmastenol; $\Delta^{7}-A v$, $\Delta^{7}$-avenasterol.

$b_{\text {tr, trace: }}<0.1 \%$.

In conclusion, the results obtained in this work suggest that maturity groups do not appear to affect the differences in chemical composition of soybeans analyzed. Although statistically significant differences exist between genotypes, when mean values in each maturity group are considered for all parameters evaluated, only oil content and oleic acid percentage show significant variations.

\section{ACKNOWLEDGEMENTS}

Cooperative investigations between Instituto de Ciencias y Tecnología de los Alimentos (ICTA -
FCEFyN - UNC) and EEA - INTA (Manfredi, Córdoba). This research was supported by grants from CONICET and CONICOR.

\section{REFERENCES}

AOAC (1980).- - Official Methods of Analysis of the Association of Official Analytical Chemists".- Horwitz, W. (Ed.). Washigton D.C., USA.

Breene, W.M., Lin, S., Hardman, L. and Orf, J. (1988).«Protein and oil content of soybeans from different geographic locations".-J. Am. Oil Chem. Soc. 65 (12), 1927-1931. 
Carreras, M.E., Fuentes, E. and Guzmán, C.A. (1989)."Chemotaxonomy of seed lipids of Cucurbitaceae grown in Argentina".-Biochem. Syst. Ecol. 17 (4), 287-291.

Carver, B.F., Burton, J.W., Carter, Jr., T.E. and Wilson, R.E. (1986).- «Response to environmental variation of soybean lines selected for altered unsaturated fatty acid composition".-Crop Sci. 26, 1176-1181.

Duve, K.J. and White, P.J. (1991).- «Extraction and identification of antioxidants in oats".-J. Am. Oil Chem. Soc. 68 (6), 365-370.

Gaydou, E.M., Bianchini, J.P. and Ratovohery, J.V. (1983)._ «Triterpene alcohols, methyl sterols, sterols, and fatty acids in five Malagasy legume seed oils".-J. Agric. Food Chem. 31 833-836.

Liu, K., Orthoefer, F. and Brown, E.A. (1995).- «Association of seed size with genotypic variation in the chemical constituents of soybeans".-J. Am. Oil Chem. Soc. 72 (2), 189-192.

Maestri, D.M. and Guzmán, C.A. (1993).-«Chemical composition of tobacco seeds (Nicotiana tabacum L) from Argentina».-J. Sci. Food Agric. 61, 227-230.

Padley, F.B., Gunstone, F.D. and Harwood, J.L. (1986)."Occurrence and Characteristics of Oils and Fats" en "The Lipid Handbook», pp. 49-170.-F.D. Gunstone, J.L. Harwood and Padley, F.B. (Eds.).- Chapman and Hall, London, UK.

Schnebly, S.R. and Fehr, W.R. (1993)._-«ffect of years and planting dates on fatty acid composition of soybean genotypes».-Crop Sci. 33, 716-719.

Recibido: Agosto 1997 Aceptado: Febrero 1998 